\title{
ANALYSIS OF HALF DIALLEL CROSS FOR SOME QUANTITATIVE CHARACTERS IN RICE (ORYZA SATIVA L.)
}

\author{
M.A. El- Hity ${ }^{1}$;A.A.Elsayed ${ }^{1}$;A.A.Abd-Allah²and M.A. Abo-Zeid \\ 1- Agronomy Dept., Faculty of Agriculture, Kafrelsheikh Univ. \\ 2- RRTC, Sakha Agr. Station, ARC, Egypt
}

\begin{abstract}
The present study was carried out at the experimental farm of the Rice Research and Training Center (RRTC)., Sakha, kafrelsheikh, during 2014 and 2015 rice growing seasons to study the inheritance of some quantitative characters using a half diallel cross among eight rice parental genotypes namely; Sakha 102, Giza 179, Sakha 105, Sakha 106, GZ 8710, GZ 1368, Wab 56 and IET 1444. The parents and their $F_{1}$ crosses were evaluated under normal and stress irrigation. Days to heading, plant height, flag leaf Area, chlorophyll content, relative water content, panicle length, number of panicles/plant, 100-kernel weight, sterility percentage and grain yield/plant were estimated. Highly significant mean squares were obtained for genotypes, parents and their crosses in all studied characters. The interactions of genotypes, parents and crosses with environments (irrigation) were detected to be highly significant for studied traits. Mean squares of general combining ability (GCA) and specific combining ability (SCA) and the interaction between environment and each of GCA and SCA were highly significant for all studied characters, indicating the importance of additive and non-additive genetic variances in determining the performance of these characters. The parental genotypes; Giza 179 and GZ 8710 were good combiners for panicles numbers/plant, 100-grain weight, sterility percentage and grain yield / plant. The crosses; (GZ $1368 \times$ IET 1444), (Giza $179 \times$ GZ 8710), (Sakha $102 \times$ IET 1444) and (Giza $179 \times$ Sakha 106) were superior on the basis of specific combining ability effect.
\end{abstract}

\section{INTRODUCTION}

Rice is the world leading cereal crop for human utilization with cultivated area of almost 600 million mega grams annually (khush, 2005). The world population is expected to reach 8 billion by 2030 and rice production must be increased by $50 \%$ in order to meet the growing demand for the world (khush and Brar, 2002).

In Egypt, the average national yield level of rice should be increased by $25-30 \%$ to meet the demands of the increasing population which seems difficult considering the narrow gap between yield potential and actual yield $10 \mathrm{t} \mathrm{ha}^{-1}$ in 2008. However, among available technologies to increase yield above the present ceiling is the exploitation of 
heterosis in hybrid rice, which appears to be practical approach for Egypt (Bastawisiet al.,2005). Lake of water resources is one of the main problem of rice cultivation and production, especially during the periods of low rainfall which affect the vegetative growth rate and amount of yield (Mostajeranand Rahimi -Echi 2009 and Venuprasad et al., 2007). Combining ability refers to the ability of a genotype to transfer its desirable genes to its progenies. Combining ability analysis is the major tool to exploit phenomenon of heterosis through estimate the combining ability effects to selecting better parents and hybrids (Yan et al. 2000, El-Mowafi and Abou-Shousha 2003, El-Diasty et al. 2008 and Mirarab et al.2011).Water stress is the major environmental that constrains the productivity and stability of crops (Araus et al, 2002). It is estimated that, more than $50 \%$ of the world rice production area is affected by drought (Bouman et al, 2005).Egypt is self sufficient in rice, but due to a high population growth rate, presence of new diseases and pests, the ongoing process of climate changes would be declined to insufficient levels. The development of drought-tolerant varieties which maintain good yield under water stress condition is of major priorty for rice research for sustainable rice production (Abd ElHadi et al, 2014). The main objective of the present study is to assess genetic parameters and combining ability for agronomic, yield and yield components characters under normal as well as water stress conditions and to identify the most desirable genotypes for rice breeding program under this conditions.

\section{MATERIALS AND METHODS}

The present study was carried out at the Experimental Farm of the Rice Research and Training Center (RRTC), Sakha, kafrelsheikh, Field Crops Research Institute, ARC during 2014 and 2015 rice growing seasons. The main objective was to study the combining ability of some rice cultivars and lines in respect to some quantitative characters .These characters; days to heading, plant height, flag leaf area, chlorophyll content, relative water content, panicle length, no. of panicles/plant, 100-kernel weight, sterility, and grain yield/plant, were taken on an individual plant basis.

In 2014 season, eight rice genotypes namely Sakha102, Giza179, Sakha105, Sakha106, GZ8710, GZ1368, Wab56 and IET1444 which represented a wide range of diversity were crossed in half-diallel mating design producing $28 \mathrm{~F}_{1}$ 's. In 2015 season, the tested genotypes (eight parents and $28 \mathrm{~F}_{1}$ crosses) were planted under both normal and drought conditions (drought stress was imposed by using flush irrigation every 12 days without standing water after irrigation) in a randomized complete blocks design with three replications. Each entry was grown in one row, five meters along with $20 \mathrm{~cm}$ between rows and 
comprised 25 hills each of a single plant. All agronomic practices were done as usual recommended during growing seasons of the study. Before one proceeds with the computation of the combined experiments, it is necessary to determine whether the error variances of the tests are homogenous or not. The test described by Bartlett (1937) is the most widely method used. The combined analysis was calculated over the two environments in the case of homogeneity error variance to test the interaction of the different genetic components with the two different environmental conditions. Data were analyzed to test the significance of the different genotypes, the analysis of variance was calculated for each character according to Steel and Torrie (1980). In case of significance, the differences among genotypes were further partitioned to GCA and SCA following Griffing 1956 (Method-2 Model1 ) as a fixed mode. Variance due to general and specific combining abilities were estimated, then $\left(g_{i}\right)$ and $\left(\overline{\mathbf{S}}_{\mathrm{ij}}\right)$ were calculated.

\section{Analysis of variance}

\section{RESULTS AND DISCUSSION}

Tables (1, 2, 3 and 4) present the mean square values among $F_{1}$ hybrids and the partitioning of genetic variances into general and specific combining abilities. Highly significant mean squares of irrigation used an environments for all studied traits, which might indicate that the differences between the two irrigation regimes were cleaned. The data revealed that there were highly significant differences among the genotypes studied for all studied traits, indicating wide diversity between them under both normal and drought conditions and their combined data.

Highly significant mean square of parents indicating the presence of differences between parents for all studied traits in both environments, which indicated wide diversity between the parents used and refer to the successful of the crossing process (Tables 1 and 2).

Highly significant mean squares were observed between crosses for all studied traits in both environments, which might indicate the great variation between these crosses due to the diversity of the parents used (Tables1 and 2).

Mean squares of the interaction between genotypes and irrigations were highly significant for the traits in view, indicating that the genotypes performed in different way from normal to stress conditions. Moreover, the parents $\times$ irrigation interaction mean squares were highly significant for all traits in consideration, meaning that the performance of each parent would be changed with the changing irrigation from normal to stress conditions, the same conclusion would 
be applicable with respect to crosses, where the crosses $\times$ irrigation interaction mean squares noted to be highly significant (Table1 and 2)

The data listed in tables 3 and 4 indicated that, there were highly significant estimates for both general and specific combining ability mean squares under the two environments and their combined data, indicating the relative importance of both additive and nonadditive genetic variances for all studied traits.

General combining ability $\times$ irrigation interaction mean squares were highly significant for all traits in question, indicating that additive gene action could be changed from environment to another. The same conclusion might be applicable with respect to non-additive gene effects, where the interaction between SCA $\times$ I mean squares were highly significant.

The relative importance of each variance was determined using the ratio of GCA/SCA mean square. The ratio of GCA/SCA mean squares were found to be greater than unity for all studied traits under both environments and their combined data. This might indicated that additive gene action was more important than non-additive one in the inheritance of the studied traits. Therefore, it could be concluded that selection procedures based on accumulation of additive effect would be successful in early segregating generation to improve these traits. similar results were obtained by Abd Allah (2004), Abd El-lateef (2006), Gaballa (2009), Hadifa (2012) and El-Hity et al., (2015).

Table (1): Mean squares of the genotypes for vegetative traits under normal and drought conditions and their combined data

\begin{tabular}{|c|c|c|c|c|c|c|c|c|c|c|c|}
\hline \multirow{2}{*}{ s.o.v } & \multicolumn{2}{|c|}{ d.f } & \multicolumn{3}{|c|}{ Days to heading } & \multicolumn{3}{|c|}{ Plant Height } & \multicolumn{3}{|c|}{ Flag Leaf Area } \\
\hline & Single & Comb & $\mathbf{N}$ & $\mathrm{s}$ & comb & $\mathbf{N}$ & $\mathrm{s}$ & comb & $\mathrm{N}$ & $\mathrm{s}$ & comb \\
\hline Replications & 2 & $\ldots .$. & 3.9 & 4.71 & & 3.1 & 5.93 & & 0.47 & 0.57 & \\
\hline Irrigation (I) & $\ldots .$. & 1 & ...... & $\ldots$ & $313.84^{\star \star}$ & $\ldots .$. & $\ldots$ & $4127.08^{\star \star}$ & ..... & $\ldots$ & $1702.05^{\star \star}$ \\
\hline $\begin{array}{l}\text { Reps/irrigation } \\
\text { (I) }\end{array}$ & ..... & 4 & $\cdots$ & $\cdots$ & 4.31 & $\ldots$ & $\ldots$ & 4.51 & $\cdots$ & $\cdots$ & 0.52 \\
\hline Genotypes (G) & 35 & 35 & $304.35^{\star \star}$ & $288.52^{\star \star}$ & $566.69^{\star \star}$ & $188.05^{* \star}$ & $73.92^{\star \star}$ & $217.59^{\star \star}$ & $54.89^{\star \star}$ & $67.42^{* \star}$ & $117.92^{\star \star *}$ \\
\hline Parents $(\mathrm{P})$ & 7 & 7 & $290.44^{\star \star}$ & $202.31^{\star \star}$ & $469.17^{\star \star}$ & $157.83^{\text {** }}$ & $96.45^{\star \star}$ & $236.19^{\star \star}$ & $44.71^{\star \star}$ & $86.08^{\star \star}$ & $120.67^{\star \star}$ \\
\hline Crosses (C) & 27 & 27 & $303.61^{\star \star}$ & $253.15^{\star \star}$ & $538.27^{\star \star}$ & $200.27^{\star \star \star}$ & $61.44^{\star \star}$ & $219.77^{\star \star}$ & $56.01^{\star \star}$ & $61.97^{\star \star}$ & $114.92^{\star \star}$ \\
\hline Pvs. .C & 1 & 1 & $421.53^{\star \star}$ & $1847.06^{* \star}$ & $2016.68^{\star \star \star}$ & $69.83^{\star \star}$ & $253.23^{* \star}$ & $28.55^{\star}$ & $95.86^{* \star}$ & $83.8^{* *}$ & $179.45^{* \star}$ \\
\hline$G \times 1$ & $\ldots \ldots$ & 35 & $\ldots$. & $\cdots$ & $26.17^{\star \star}$ & $\ldots$. & $\ldots$ & $44.38^{\star \star}$ & $\ldots$. & $\ldots$ & $4.39^{\star \star}$ \\
\hline$P \times 1$ & $\ldots . .$. & 7 & $\ldots$ & $\ldots$ & $23.58^{\star \star \star}$ & $\ldots$. & $\ldots$ & $18.09^{* \star}$ & $\ldots$ & $\ldots$ & $10.12^{\star \star *}$ \\
\hline$C \times 1$ & $\ldots . .$. & 27 & $\ldots .$. & $\ldots$ & $18.49^{\star \star \star}$ & $\ldots .$. & $\ldots$ & $41.93^{* \star}$ & $\ldots .$. & $\ldots$ & $3.06^{* *}$ \\
\hline Pvs. .C $\times 1$ & $\ldots .$. & 1 & $\ldots .$. & $\ldots$. & $251.92^{\star \star}$ & $\ldots .$. & $\ldots$. & $294.5^{\star \star}$ & $\ldots .$. & $\ldots$ & $2.9^{* *}$ \\
\hline Error & 70 & 140 & 7.21 & 7.06 & 7.13 & 6.72 & 6.48 & 6.6 & 0.94 & 1.02 & 0.98 \\
\hline \multirow{2}{*}{ s. o.v } & \multicolumn{2}{|c|}{ d.f } & \multicolumn{3}{|c|}{ Chlorophyll content } & \multicolumn{3}{|c|}{ 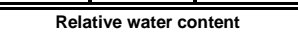 } & & & \\
\hline & Single & Comb & $\mathbf{N}$ & $\mathrm{s}$ & comb & $\mathbf{N}$ & $\mathrm{s}$ & Comb & & & \\
\hline Replications & 2 & $\ldots .$. & 0.05 & 1.3 & & 1.33 & 0.12 & & & & \\
\hline Irrigation (I) & 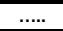 & 1 & $\ldots$. & $\ldots$ & $942.38^{\star \star}$ & $\ldots .$. & $\ldots$ & $1232.5^{\star \star}$ & & & \\
\hline $\begin{array}{c}\text { Reps/irrigation } \\
\text { (I) }\end{array}$ & $\cdots \cdot$. & 4 & $\cdots$ & $\cdots$ & 0.67 & $\cdots$ & $\cdots$ & 0.72 & & & \\
\hline Genotypes (G) & 35 & 35 & $32.96^{\star \star}$ & $27.27^{\star \star}$ & $51.78^{\star \star}$ & $96.39^{\star \star}$ & $90.45^{\star \star}$ & $178.94^{\star \star}$ & & & \\
\hline Parents $(\mathrm{P})$ & 7 & 7 & $13.52^{\star \star}$ & $17.04^{\star \star}$ & $23.7^{\star \star \star}$ & $79.39^{\star \star \star}$ & $97.97^{\star \star}$ & $169.96^{\star \star}$ & & & \\
\hline Crosses (C) & 27 & 27 & $35.84^{\star \star}$ & $30.05^{\star \star}$ & $57.12^{\star \star}$ & $103.93^{\star \star}$ & $89.93^{\star \star}$ & $185.79^{\star \star}$ & & & \\
\hline Pvs. .C & 1 & 1 & $91.25^{\star \star}$ & $23.77^{\star \star}$ & $104.08^{\star \star}$ & $12.03^{\star \star}$ & $51.89^{\star \star}$ & $56.94^{\star \star}$ & & & \\
\hline$G \times 1$ & $\ldots$ & 35 & $\ldots$. & $\ldots$ & $8.45^{\star \star}$ & $\ldots$. & $\ldots$ & $7.9^{\star \star}$ & & & \\
\hline$P \times 1$ & $\ldots . .$. & 7 & $\ldots$ & $\ldots$ & $6.86^{*}$ & $\ldots$ & $\ldots$ & $7.4^{\star \star}$ & & & \\
\hline $\mathrm{C} \times \mathrm{I}$ & $\ldots . .$. & 27 & ...... & $\ldots .$. & $8.77^{\star \star}$ & $\ldots .$. & $\ldots$ & $8.07^{\star \star \hbar}$ & & & \\
\hline
\end{tabular}


\begin{tabular}{|c|c|c|c|c|c|c|c|c||} 
P vs. .C $\times 1$ & $\ldots .$. & 1 & $\ldots .$. & $\ldots$. & $10.94^{\star *}$ & $\ldots .$. & $\ldots$. & $6.98^{\star *}$ \\
\hline Error & 70 & 140 & 2.89 & 3.42 & 3.15 & 1.78 & 2.05 & 1.91 \\
\hline
\end{tabular}

* and ${ }^{* *}$ significant at 0.05 and 0.01 levels of probability, respectively.

$\mathrm{N}$ : normal condition. S: drought condition. Comb: combined data.

Table (2): Mean squares of the genotypes for yield and its components under normal and drought conditions and their combined data

\begin{tabular}{|c|c|c|c|c|c|c|c|c|c|c|c|}
\hline \multirow{2}{*}{ s.o.v } & \multicolumn{2}{|c|}{ d.f } & \multicolumn{3}{|c|}{ Panicle length } & \multicolumn{3}{|c|}{ No of Panicles / plant } & \multicolumn{3}{|c|}{ 100-kernel Weight } \\
\hline & Single & Comb & $\mathbf{N}$ & $\mathrm{s}$ & comb & $\mathbf{N}$ & $\mathrm{s}$ & comb & $\mathbf{N}$ & $\mathrm{s}$ & comb \\
\hline Replications & 2 & $\ldots .$. & 0.03 & 0.24 & & 0.8 & 0.2 & & 0.03 & 0.03 & \\
\hline Irrigation (I) & $\ldots .$. & 1 & $\ldots .$. & $\ldots$ & $330.5^{\star \star}$ & $\ldots .$. & $\ldots$ & $1048.3^{\star \star}$ & $\ldots .$. & $\ldots$ & $11.59^{\star \star}$ \\
\hline $\begin{array}{l}\text { Reps/irrigation } \\
\text { (I) }\end{array}$ & ..... & 4 & .... & .... & 0.13 & .... & .... & 0.5 & .... & $\cdots$ & 0.03 \\
\hline Genotypes (G) & 35 & 35 & $14.62^{\star \star}$ & $19.14^{\star \star}$ & $30.66^{\star \star}$ & $62.16^{\star \star}$ & $32.43^{\star \star}$ & $79.62^{\star \star}$ & $0.26^{\star \star}$ & $0.38^{\star \star}$ & $0.51^{\star \star}$ \\
\hline Parents (P) & 7 & 7 & $2.49^{\text {* }}$ & $2.60^{\star \star *}$ & $2.33^{* \star}$ & $59.1^{\star \star}$ & $36.07^{\star \star}$ & $71.92^{\star \star}$ & $0.15^{* \star}$ & $0.23^{* \star}$ & $0.24^{\star \star}$ \\
\hline Crosses (C) & 27 & 27 & $16.42^{\star \star}$ & $17.81^{\star \star}$ & $31.57^{\star \star}$ & $64.6^{* \star}$ & $26.39^{\star \star}$ & $83.12^{\star \star}$ & $0.28^{\star \star}$ & $0.43^{\text {** }}$ & $0.57^{\star \star}$ \\
\hline Pvs. .C & 1 & 1 & $51.06^{\star \star \star}$ & $170.9^{\star \star}$ & $204.39^{\star \star}$ & $17.57^{\star \star}$ & $169.81^{\star \star}$ & $39.07^{\star \star}$ & $0.61^{\star \star}$ & $0.17^{\star \star}$ & $0.72^{\star \star}$ \\
\hline$G \times I$ & $\ldots .$. & 35 & $\ldots .$. & $\ldots$ & $3.11^{\star \star}$ & $\ldots .$. & $\ldots$ & $14.96^{\star \star}$ & $\ldots .$. & $\ldots$ & $0.141^{\star \star}$ \\
\hline$P \times 1$ & $\ldots .$. & 7 & $\ldots$ & $\ldots$ & $2.76^{\star \star}$ & $\ldots$ & $\ldots$ & $23.25^{\star \star}$ & $\ldots$ & $\ldots$ & $0.142^{\star \star}$ \\
\hline$C \times 1$ & $\ldots .$. & 27 & $\ldots .$. & $\ldots$ & $2.66^{\star \star}$ & $\ldots .$. & $\ldots$ & $7.87^{\text {tᄎ }}$ & ..... & $\ldots$ & $0.143^{* \star}$ \\
\hline Pvs. . $\mathrm{C} \times \mathrm{I}$ & $\ldots .$. & 1 & $\ldots .$. & $\ldots$. & $17.57^{\star \star}$ & $\ldots . .$. & $\ldots$. & $148.31^{\star \star}$ & $\ldots . .$. & $\ldots$ & 0.07 \\
\hline Error & 70 & 140 & 0.87 & 0.93 & 0.9 & 2.12 & 1.45 & 1.79 & 0.019 & 0.017 & 0.018 \\
\hline \multirow{2}{*}{ s. o.v } & \multicolumn{2}{|c|}{ d.f } & \multicolumn{3}{|c|}{ Sterility \% } & \multicolumn{3}{|c|}{ Grain yield/plant } & & & \\
\hline & Single & Comb & $\mathrm{N}$ & $\mathrm{s}$ & comb & $\mathbf{N}$ & $\mathrm{s}$ & Comb & & & \\
\hline Replications & 2 & ..... & 0.12 & 0.94 & & 1.23 & 3.91 & & & & \\
\hline Irrigation (I) & $\ldots .$. & 1 & $\ldots .$. & $\ldots$. & $3148.6^{\star \star}$ & ...... & $\ldots$. & $21009.89^{* \star}$ & & & \\
\hline $\begin{array}{c}\text { Reps/irrigation } \\
\text { (I) }\end{array}$ & $\ldots .$. & 4 & $\ldots$. & $\ldots$. & 0.53 & $\ldots$. & $\ldots$. & 2.57 & & & \\
\hline Genotypes (G) & 35 & 35 & $345.39^{\star \star}$ & $500.14^{\star \star}$ & $802.85^{\star \star}$ & $90.35^{\star \star}$ & $88.93^{* \star}$ & $165.32^{\star \star}$ & & & \\
\hline Parents $(\mathbf{P})$ & 7 & 7 & $12.88^{\text {t* }}$ & $59.77^{\star \star}$ & $49.45^{\star \star}$ & $14.71^{\star \star}$ & $89.58^{\star \star}$ & $70^{\star \star}$ & & & \\
\hline Crosses (C) & 27 & 27 & $357.83^{\star \star}$ & $530.18^{\star \star}$ & $839.05^{\star \star}$ & $88.9^{* \star}$ & $82.61^{\star \star}$ & $164.05^{\star \star}$ & & & \\
\hline Pvs. .C & 1 & 1 & $2337.09^{\star \star}$ & $2771.68^{\star \star}$ & $5099.52^{\star \star}$ & $658.84^{\star \star}$ & $254.98^{* \star}$ & $866.78^{\star \star}$ & & & \\
\hline$G \times I$ & $\ldots .$. & 35 & $\ldots .$. & $\ldots$ & $42.68^{\star \star}$ & $\ldots .$. & $\ldots$. & $13.96^{\star \star}$ & & & \\
\hline$P \times 1$ & $\ldots .$. & 7 & $\ldots$. & $\ldots$. & $23.2^{\star \star}$ & $\ldots$ & $\ldots$. & $34.3^{\text {t* }}$ & & & \\
\hline $\mathrm{C} \times \mathrm{I}$ & $\ldots .$. & 27 & $\ldots$. & $\ldots$ & $48.96^{\star \star *}$ & $\ldots .$. & $\ldots$ & $7.46^{\star}$ & & & \\
\hline P vs. .C $\times I$ & $\ldots .$. & 1 & $\ldots .$. & $\ldots$. & 9.26 & $\ldots .$. & $\ldots$ & $47.04^{\star \star}$ & & & \\
\hline Error & 70 & 140 & 3.92 & 4.68 & 4.3 & 4.56 & 4.7 & 4.63 & & & \\
\hline
\end{tabular}

${ }^{*}$ and ${ }^{* *}$ significant at 0.05 and 0.01 levels of probability, respectively.

$\mathrm{N}$ : normal condition. S: drought condition. Comb: combined data.

Table (3): Mean squares of general combining ability (GCA) and specific combining ability (SCA) and their interaction under normal and drought conditions and their combined data for agronomic traits

\begin{tabular}{|c|c|c|c|c|c|c|c|c|c|c|c|}
\hline \multirow{2}{*}{ s.o.v } & \multicolumn{2}{|c|}{ d.f } & \multicolumn{3}{|c|}{ Days to heading } & \multicolumn{3}{|c|}{ Plant Height } & \multicolumn{3}{|c|}{ Flag Leaf Area } \\
\hline & Single & Comb & $\mathbf{N}$ & $\mathrm{s}$ & Comb & $\mathbf{N}$ & $\mathrm{s}$ & Comb & $\mathbf{N}$ & $\mathrm{s}$ & Comb \\
\hline Genotypes (G) & 35 & 35 & $304.35^{\star \star}$ & $288.52^{\star \star}$ & $566.69^{\star \star}$ & $188.05^{\star \star}$ & $73.92^{\star \star}$ & $217.59^{\star \star}$ & $54.89^{* \star}$ & $67.42^{\star \star}$ & $117.92^{\star \star *}$ \\
\hline G.C.A & 7 & 7 & $118.23^{\text {** }}$ & $92.67^{\star \star}$ & $207.43^{\star \star}$ & $212.74^{\star \star}$ & $59.24^{\star \star \star}$ & $242.34^{\star \star}$ & $70.11^{\star \star}$ & $89.93^{\text {th }}$ & $159.14^{* *}$ \\
\hline S.C.A & 28 & 28 & $97.25^{\star \star}$ & $90.05^{\star \star}$ & $184.26^{\star \star}$ & $25.17^{\star \star}$ & $15.99^{\star \star}$ & $30.08^{\star \star}$ & $5.34^{* \star}$ & $5.61^{\star \star}$ & $9.35^{\star \star}$ \\
\hline$G \times 1$ & $\ldots .$. & 35 & $\ldots .$. & & $26.17^{\star \star}$ & & & $44.38^{* \star}$ & & & $4.39^{\star \star}$ \\
\hline G.C.A $\times 1$ & $\ldots .$. & 7 & $\ldots .$. & $\ldots .$. & $13.47^{\star \star}$ & $\ldots \ldots$ & $\ldots .$. & $29.65^{\star \star}$ & $\ldots .$. & $\ldots .$. & $1.89^{* \star}$ \\
\hline S.C.A $\times 1$ & & 28 & & & $10.04^{\star \star}$ & & & $11.08^{\star \star}$ & & & $1.61^{\star \star}$ \\
\hline Error & 70 & 140 & 2.4 & 2.35 & 2.38 & 2.24 & 2.16 & 2.2 & 0.31 & 0.34 & 0.33 \\
\hline G.C.A/S.C.A & $\ldots .$. & $\ldots .$. & 1.22 & 1.03 & 1.13 & 8.45 & 3.71 & 8.06 & 13.12 & 16.03 & 17.03 \\
\hline $\begin{array}{c}\text { G.C.A } \times 1 / \text { S.C.A } \\
\times 1\end{array}$ & $\cdots \cdots$ & ...... & $\ldots \ldots$ & $\ldots$. & 1.34 & $\ldots$. & $\ldots$. & 2.68 & ..... & $\cdots \cdots$ & 1.17 \\
\hline sov & & & & rophyll ce & & & ve water & tent & & & \\
\hline & Single & Comb & $\bar{N}$ & $\mathrm{~s}$ & Comb & $\overline{\mathbf{N}}$ & $\mathrm{s}$ & Comb & & & \\
\hline Genotypes (G) & 35 & 35 & $32.96^{* \star}$ & $27.27^{\star \star}$ & $51.78^{\star \star}$ & $96.39^{\star \star}$ & $90.45^{\star \star}$ & $178.94^{\star \star}$ & & & \\
\hline G.C.A & 7 & 7 & $21.72^{* \star}$ & $19.02^{\star \star}$ & $34.53^{\star \star}$ & $92.57^{\star \star}$ & $85.37^{* \star}$ & $176.28^{\star \star}$ & & & \\
\hline S.C.A & 28 & 28 & $8.3^{* \star}$ & $6.61^{\star \star}$ & $12.94^{\star \star}$ & $17.02^{\star \star}$ & $16.35^{\star \star}$ & $30.49^{\star \star}$ & & & \\
\hline$G \times 1$ & $\ldots . .$. & 35 & $\ldots .$. & $\ldots$. & $8.45^{\star \star}$ & $\ldots .$. & $\ldots$. & $7.9^{* \star}$ & & & \\
\hline G.C.A $\times 1$ & $\ldots .$. & 7 & $\cdots$ & $\ldots .$. & $6.21^{\star \star}$ & $\ldots .$. & $\ldots$. & $4.66^{* \star}$ & & & \\
\hline S.C.A $A$ I & $\ldots .$. & 28 & & & $1.97^{* \star}$ & & & $2.88^{\star \star}$ & & & \\
\hline Error & 70 & 140 & 0.96 & 1.14 & 1.05 & 0.59 & 0.68 & 0.64 & & & \\
\hline G.C.A/S.C.A & $\ldots .$. & $\ldots .$. & 2.62 & 2.88 & 2.67 & 5.44 & 5.22 & 5.78 & & & \\
\hline $\begin{array}{c}\text { G.C.A } \times \text { I/ S.C.A } \\
\times 1\end{array}$ & ..... & ..... & $\ldots$ & ..... & 3.15 & ..... & ..... & 1.61 & & & \\
\hline
\end{tabular}


Table (4): Mean squares of general combining ability (GCA) and specific combining ability (SCA) and their interaction under normal and drought conditions and their combined data for yield and its components.

\begin{tabular}{|c|c|c|c|c|c|c|c|c|c|c|c|}
\hline \multirow{2}{*}{ s. o. v } & \multicolumn{2}{|c|}{ d.f } & \multicolumn{3}{|c|}{ Panicle length } & \multicolumn{3}{|c|}{ No of Panicles / plant } & \multicolumn{3}{|c|}{ 100-kernel Weight } \\
\hline & Single & Comb & $\bar{N}$ & $\mathrm{~s}$ & Comb & $\bar{N}$ & $\mathrm{~s}$ & Comb & $\mathbf{N}$ & $\mathrm{s}$ & Comb \\
\hline Genotypes (G) & 35 & 35 & $14.62^{\star \star}$ & $19.14^{* *}$ & $30.66^{* *}$ & $62.16^{* \star}$ & $32.43^{* *}$ & $79.62^{\star \star}$ & $0.26^{* \star}$ & $0.38^{* *}$ & $0.51^{* *}$ \\
\hline G.C.A & 7 & $\frac{7}{7}$ & $11.31^{\star \star}$ & $10.39^{\star \star \star}$ & $21.36^{* *}$ & $48.68^{* \star}$ & $14.86^{* *}$ & $54.1^{\star \star *}$ & $0.24^{\star \star}$ & $0.4^{* *}$ & $0.54^{\star \star}$ \\
\hline S.C.A & 28 & 28 & $3.27^{* *}$ & $5.38^{* \star}$ & $7.43^{* *}$ & $\frac{10.00}{13.73^{* *}}$ & $9.8^{* *}$ & $19.65^{* *}$ & $0.05^{* *}$ & $0.06^{\star \star}$ & $0.08^{\star \star}$ \\
\hline$G \times I$ & $\ldots . .$. & 35 & $\ldots . .$. & $\ldots .$. & $3.11^{\star \star}$ & $\ldots .$. & $\ldots$. & $14.96^{* *}$ & $\ldots .$. & $\ldots$. & $0.14^{\star \star}$ \\
\hline G.C.A $\times 1$ & $\cdots$ & 7 & & & $0.82^{\star \star}$ & & & $9.44^{* \star}$ & & & $0.1^{\text {t* }}$ \\
\hline S.C.A $\times 1$ & $\cdots$ & 28 & $\cdots$ & $\ldots . .$. & 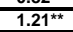 & $\cdots$ & $\ldots . .$. & $3.87^{* *}$ & $\ldots .$. & & $0.03^{* *}$ \\
\hline Error & 70 & 140 & 0.29 & 0.31 & 0.3 & 0.71 & 0.48 & 0.6 & 0.0062 & 0.0056 & 0.0059 \\
\hline G.C.A/ S.C.A & .... & .... & 3.46 & 1.93 & 2.87 & 3.55 & 1.52 & 2.75 & 4.65 & 6.7 & 7.08 \\
\hline $\begin{array}{c}\text { G.C.A } \times 1 / \text { S.C.A } \\
\times 1\end{array}$ & ...... & ...... & $\cdots .$. & $\ldots .$. & 0.68 & $\ldots .$. & $\ldots .$. & 2.43 & $\ldots .$. & $\ldots .$. & 3.33 \\
\hline $50 y$ & & & & $\overline{\text { Sterility } 9}$ & & & in yield/ $/ \mathrm{s}$ & & & & \\
\hline & Single & Comb & $\bar{N}$ & $\mathrm{~s}$ & Comb & $\bar{N}$ & $\mathrm{~s}$ & Comb & & & \\
\hline Genotypes (G) & 35 & $\frac{35}{35}$ & $345.39^{* \star *}$ & $500.14^{\star \star}$ & $802.85^{* * 4}$ & $90.35^{\star \star}$ & $88.93^{* *}$ & $165.32^{\star \star 4}$ & & & \\
\hline G.C.A & 7 & 7 & $120.31^{\star \star}$ & $184.92^{\star \star}$ & $277.65^{* *}$ & $52.61^{\star \star}$ & $92.82^{\star \star *}$ & $139.83^{\text {t* }}$ & & & \\
\hline S.C.A & 28 & 28 & $116.08^{\text {t* }}$ & $162.16^{* \star}$ & $265.11^{* \star}$ & $24.49^{* *}$ & $13.85^{* *}$ & $33.92^{* \star}$ & & & \\
\hline$G \times 1$ & $\ldots$ & 35 & $\ldots .0$ & $\ldots$. & $42.68^{*+4}$ & 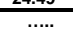 & $\ldots . .$. & $\frac{0.066^{* * *}}{13.96^{* *}}$ & & & \\
\hline G.C.A $\times 1$ & $\ldots .$. & 7 & $\ldots .$. & $\ldots \ldots$ & $18.58^{* *}$ & $\ldots \ldots$ & $\ldots .$. & $5.59^{* *}$ & & & \\
\hline S.C.A .1 & & 28 & & & $\frac{10.00}{13.14^{* *}}$ & & & $4.42^{*+*}$ & & & \\
\hline Error & 70 & 140 & 1.31 & 1.56 & 1.43 & 1.52 & 1.57 & 1.54 & & & \\
\hline G.C.A/S.C.A & $\ldots .$. & $\ldots .$. & 1.03 & 1.14 & 1.05 & 2.15 & 6.7 & 4.12 & & & \\
\hline $\begin{array}{l}\text { G.C.A } \times \text { I/ S.C.A } \\
\times 1\end{array}$ & ..... & ..... & ..... & ...... & 1.41 & ...... & .... & 1.26 & & & \\
\hline
\end{tabular}

${ }^{*}$ and ${ }^{* *}$ significant at 0.05 and 0.01 levels of probability, respectively.

$\mathrm{N}$ : normal condition. S: drought condition. Comb: combined data.

However, it could be detected that the ratio of GCA/SCA mean squares were equal one for no. of days to heading at stress condition, sterility percentage at normal condition and the combined analysis, which might indicate that both additive and non-additive gene effects played the same role with respect to the inheritance of these cases.

The ratio of GCA $\times$ I / SCA $\times$ I mean squares were more than unity for all studied traits, except with panicle length. This might indicate that, additive gene action was more interacted with both environments than non-additive one for these traits, while for panicle length where the same ratio was less than unity, which might indicated the non-additive gene effects were more interacted with both environments than additive one.

\section{General combining ability effects:}

Estimates of general combining ability effects $\left(\hat{g_{j}}\right)$ for individual parental genotypes for all studied traits at both environments and their combined data are presented in Tables (5 and 6).

Concerning days to heading the parents; Sakha 105, Sakha 106 and GZ 8710 showed highly significant in negative direction general combining ability effects at the two environments and their combined data, indicating that these genotypes could be considered as good combiners for earliness. Among the previous three parents (Sakha 105) was the best combiner as it gave the highest negative value under both environments and their combined data. 
Regarding plant height, the parents; Giza 179 and Sakha 105 showed highly significant desirable $(\hat{g})$ in both environments and their combined data, proving to be excellent parents for developing short stature genotypes under drought stress as well as at normal irrigation. For flag leaf area, the parents; GZ1368, Wab 56 and IET 1444 showed highly significant positive ( $\left(\hat{g_{i}}\right)$ at the two conditions and their combined data, proving to be good combiners for this trait.

The rice parental genotypes; GZ1368, Wab 56 and IET 1444 showed significant positive ( $\left.\hat{g}_{j}\right)$ in the two environments and their combined data, proving to be good combiners for chlorophyll content.

With respect to relative water content, the parents; Giza179, IET 1444 ,GZ 1368 and Wab 56 showed highly significant positive $\left(\widehat{g_{i}}\right)$ at both environments and their combined data. These results indicated that, these parents could be considered as good combines for this trait.

For panicle length, significant positive $\left(\hat{g_{i}}\right)$ was obtained from

Sakha 102 and Giza 179, indicating that these rice parental genotypes could be used as good combiners under normal as well as drought stress in breeding program.

Concerning panicles number / plant, the parental genotypes; Giza 179, GZ 8710 and IET 1444 showed highly significant positive $\left(\hat{g_{i}}\right)$ at two environments and their combined data, proving to be good combiners for number of panicles / plant.

Regarding 100-kernal weight, the parental genotypes; Sakha 102, Giza 179 and GZ 8710 could be considered as good combiners where they had significant $\left(\hat{g}_{i}\right)$ under both environments and their combined data.

With respect to sterility percentage, the parents; Giza 179, Wab 56, GZ 28710 and IET 1444 give highly significant negative $\left(\hat{g_{1}}\right)$ under both environments and their combined data, proving to be good combiners for improving this trait. 
Table (5): Estimates of general combining ability effects of the parental genotypes under normal and drought conditions and their combined datafor vegetative traits

\begin{tabular}{|c|c|c|c|c|c|c|c|c|c|c|c|c|c|c|c|}
\hline \multirow{2}{*}{ Parents } & \multicolumn{3}{|c|}{ Days to heading (day) } & \multicolumn{3}{|c|}{ Plant height $(\mathrm{cm})$} & \multicolumn{3}{|c|}{ Flag leaf area $\left(\mathrm{cm}^{2}\right)$} & \multicolumn{3}{|c|}{ Chlorophyll content $(\mathrm{mg} / \mathrm{g})$} & \multicolumn{3}{|c|}{ Relative water content } \\
\hline & $\mathbf{N}$ & s & Comb & $\mathrm{N}$ & s & Comb & $\mathbf{N}$ & s & Comb & $\mathrm{N}$ & s & Comb & $\mathrm{N}$ & s & comb \\
\hline $\begin{array}{c}\text { P1 (Sakha } \\
\text { 102) }\end{array}$ & -0.57 & $-1.1^{\star}$ & $-0.83^{\star \star}$ & $4.44^{\star \star}$ & $1.54^{\star \star}$ & $2.99^{\star \star}$ & $-3.17^{\star \star}$ & $-3.66^{\star \star}$ & $-3.41^{\star \star}$ & $-1.57^{\star \star}$ & 0.2 & $-0.69^{\star \star}$ & $-2.89^{\star \star}$ & $-2.53^{\star \star}$ & $-2.71^{\star \star}$ \\
\hline $\begin{array}{c}\text { P2 (Giza } \\
\text { 179) }\end{array}$ & $4.02^{\star \star}$ & $3.89^{\star \star}$ & $3.95^{\star \star}$ & $-5.6^{\star \star}$ & $-2.86^{\star \star}$ & $-4.23^{\star \star}$ & 0.01 & $0.54^{\star \star}$ & $0.27^{\star \star}$ & $-1.2^{\star \star}$ & -0.21 & $-0.7^{\star \star}$ & $2.61^{\star \star}$ & $1.82^{\star \star}$ & $2.21^{\star \star}$ \\
\hline $\begin{array}{c}\text { P3 (Sakha } \\
\text { 105) }\end{array}$ & $-4.48^{\star \star}$ & $-4.18^{\star \star}$ & $-4.33^{\star \star}$ & $-5.6^{\star \star}$ & $-3.62^{\star \star}$ & $-4.61^{\star \star}$ & $-3.36^{\star \star}$ & $-4.04^{\star \star}$ & $-3.7^{\star \star}$ & 0.21 & -0.16 & 0.03 & $-4.71^{\star \star}$ & $-4.58^{\star \star}$ & $-4.65^{\star \star}$ \\
\hline $\begin{array}{c}\text { P4 (Sakha } \\
\text { 106) }\end{array}$ & $-3.21^{\star \star}$ & $-3.53^{\star \star}$ & $-3.37^{\star \star}$ & $1.9^{\star \star}$ & 0.75 & $1.32^{\star \star}$ & $-1.42^{\star \star}$ & $-1.48^{\star \star}$ & $-1.45^{\star \star}$ & $-0.61^{\star}$ & $-2.22^{\star \star}$ & $-1.41^{\star \star}$ & $-1.13^{\star \star}$ & $-1.76^{\star \star}$ & $-1.45^{\star \star}$ \\
\hline $\begin{array}{c}\text { P5 (GZ } \\
8710)\end{array}$ & $-3.38^{\star \star}$ & $-1.55^{\star \star}$ & $-2.46^{\star \star}$ & $-4.37^{\star \star}$ & -0.74 & $-2.55^{\star \star}$ & 0.17 & 0.16 & $0.16^{\star}$ & $-1.53^{\star \star}$ & $-1.47^{\star \star}$ & $-1.5^{\star \star}$ & $-0.62^{\star \star}$ & 0.45 & -0.09 \\
\hline $\begin{array}{c}\text { P6 (GZ } \\
1368)\end{array}$ & $1.02^{\star}$ & $1.11^{\star}$ & $1.07^{\star \star}$ & $2.03^{\star \star}$ & 0.79 & $1.41^{\star \star}$ & $0.7^{\star \star}$ & $0.72^{\star \star}$ & $0.71^{\star \star}$ & $2.2^{\star \star}$ & $0.89^{\star \star}$ & $1.54^{\star \star}$ & $1.16^{\star \star}$ & $1.07^{\star \star}$ & $1.11^{\star \star}$ \\
\hline $\begin{array}{c}\text { P7(Wab } \\
56)\end{array}$ & $4.22^{\star \star}$ & $3.27^{\star \star}$ & $3.74^{\star \star}$ & $6.23^{\star \star}$ & $4.01^{\star \star}$ & $5.12^{\star \star}$ & $3.87^{\star \star}$ & $4.14^{\star \star}$ & $4.01^{\star \star}$ & $0.72^{\star}$ & $0.8^{\star}$ & $0.76^{\star \star}$ & $0.7^{\star \star}$ & $0.62^{\star}$ & $0.66^{\star \star}$ \\
\hline $\begin{array}{l}\text { P8 (IET } \\
1444) \\
\end{array}$ & $2.37^{\star \star}$ & $2.08^{\star \star}$ & $2.23^{\star \star}$ & $0.96^{\star}$ & 0.13 & $0.55^{\star \star}$ & $3.2^{\star \star}$ & $3.62^{\star \star}$ & $3.41^{\star \star}$ & $1.79^{\star \star}$ & $2.17^{\star \star}$ & $1.98^{\star \star}$ & $4.89^{\star \star}$ & $4.92^{\star \star}$ & $4.91^{\star \star}$ \\
\hline $\begin{array}{l}\text { L.S.D.05 } \\
\text { (gi) }\end{array}$ & 0.91 & 0.91 & 0.36 & 0.88 & 0.87 & 0.34 & 0.33 & 0.34 & 0.13 & 0.58 & 0.63 & 0.24 & 0.45 & 0.49 & 0.18 \\
\hline $\begin{array}{l}\text { L.S.D.01 } \\
\text { (gi) }\end{array}$ & 1.21 & 1.20 & 0.47 & 1.17 & 1.15 & 0.46 & 0.44 & 0.46 & 0.18 & 0.77 & 0.83 & 0.32 & 0.60 & 0.64 & 0.25 \\
\hline $\begin{array}{c}\text { L.S.D } \\
.05(\mathrm{gi}-\mathrm{gj})\end{array}$ & 1.38 & 1.37 & 0.68 & 1.33 & 1.31 & 0.65 & 0.50 & 0.52 & 0.25 & 0.88 & 0.95 & 0.45 & 0.69 & 0.74 & 0.35 \\
\hline $\begin{array}{c}\text { L.S.D } \\
.01(\mathrm{gi}-\mathrm{gj})\end{array}$ & 1.83 & 1.81 & 0.90 & 1.77 & 1.73 & 0.87 & 0.66 & 0.69 & 0.33 & 1.16 & 1.26 & 0.60 & 0.91 & 0.97 & 0.47 \\
\hline
\end{tabular}

Table (6): Estimates of general combining ability effects of the parental genotypes under normal and drought conditions and their combined datafor grain yield / plant and its components traits

\begin{tabular}{|c|c|c|c|c|c|c|c|c|c|c|c|c|c|c|c|}
\hline \multirow{2}{*}{ Parents } & \multicolumn{3}{|c|}{ Panicle length $(\mathrm{cm})$} & \multicolumn{3}{|c|}{ No of panicles / plant } & \multicolumn{3}{|c|}{ 100-kernel weight (g) } & \multicolumn{3}{|c|}{ Sterility \% } & \multicolumn{3}{|c|}{ Grain yield/plant (g) } \\
\hline & $\mathbf{N}$ & $\mathrm{s}$ & comb & $\mathbf{N}$ & $\mathrm{s}$ & Comb & $\mathbf{N}$ & s & comb & $\mathbf{N}$ & s & comb & $\mathbf{N}$ & s & Comb \\
\hline P1 (Sakha 102) & $0.83^{\star \star}$ & $1.2^{\star \star}$ & $1.02^{\star \star}$ & $-1.93^{\star \star}$ & $-1.64^{\star \star}$ & $-1.78^{\star \star}$ & $0.22^{\star \star}$ & $0.08^{\star \star}$ & $0.15^{\star \star}$ & $5.94^{\star \star}$ & $7.49^{\star \star}$ & $6.72^{\star \star}$ & $-3.46^{\star \star}$ & $-3.95^{\star \star}$ & $-3.71^{* \star}$ \\
\hline P2 (Giza 179) & $1.59^{\star \star}$ & $1.51^{\star \star}$ & $1.55^{\star \star}$ & $4.07^{\star \star}$ & $1.66^{\star \star}$ & $2.87^{\star \star}$ & $0.17^{\star \star}$ & $0.27^{\star \star}$ & $0.22^{\star \star}$ & $-3.82^{\star \star}$ & $-2.26^{\star \star}$ & $-3.04^{\star \star}$ & $3.46^{* \star}$ & $4.23^{* *}$ & $3.84^{\star \star}$ \\
\hline P3 (Sakha 105) & $-1.27^{\star \star}$ & $-0.96^{\star \star}$ & $-1.12^{\star \star}$ & $-0.93^{\star \star}$ & $-0.93^{\star \star}$ & $-0.93^{\star \star}$ & $-0.13^{\star \star}$ & $-0.26^{\star \star}$ & $-0.19^{\star \star}$ & $0.94^{\star \star}$ & $2.64^{\star \star}$ & $1.79^{\star \star}$ & $-3.05^{\star \star}$ & $-4.06^{\star \star}$ & $-3.56^{\star \star}$ \\
\hline P4 (Sakha 106) & $-1.01^{\star \star}$ & $-0.84^{\star \star}$ & $-0.93^{\star \star}$ & -0.18 & $-0.79^{\star \star}$ & $-0.48^{\star \star}$ & $-0.07^{\star \star}$ & $-0.05^{\star}$ & $-0.06^{\star \star}$ & $2.33^{\star \star}$ & $2.91^{\star \star}$ & $2.62^{\star \star}$ & -0.42 & $-1.71^{\star \star}$ & $-1.07^{\star \star}$ \\
\hline P5 (GZ 8710) & $-1.2^{\star \star}$ & $-1.33^{\star \star}$ & $-1.26^{\star \star}$ & $1.14^{\star \star}$ & $1.36^{\star \star}$ & $1.25^{\star \star}$ & $0.08^{\star \star}$ & $0.3^{* \star}$ & $0.19^{\star \star}$ & $0.72^{\star}$ & $-2.8^{\star \star}$ & $-1.04^{\star \star}$ & $1.01^{\star \star}$ & $2.35^{\star \star}$ & $1.68^{\star \star}$ \\
\hline P6 (GZ 1368) & -0.05 & -0.08 & -0.07 & $-2^{\star \star}$ & 0.15 & $-0.93^{\star \star}$ & $-0.11^{\star *}$ & $-0.15^{\star \star}$ & $-0.13^{\star \star}$ & $-0.97^{\star \star}$ & -0.17 & $-0.57^{\star \star}$ & 0.53 & -0.4 & 0.06 \\
\hline P7(Wab 56) & $0.68^{\star \star}$ & 0.31 & $0.5^{\star \star}$ & $-2.04^{\star \star}$ & $-0.79^{\star \star}$ & $-1.42^{\star \star}$ & 0.04 & $-0.11^{\star \star}$ & $-0.03^{\star \star}$ & $-0.69^{\star}$ & $-1.23^{\star \star}$ & $-0.96^{\star \star}$ & 0.65 & $1.87^{\star \star}$ & $1.26^{* \star}$ \\
\hline P8 (IET 1444) & $0.43^{\star \star}$ & 0.19 & $0.31^{\star \star}$ & $1.86^{\star \star}$ & $0.98^{\star \star}$ & $1.42^{\star \star}$ & $-0.21^{\star \star}$ & $-0.09^{\star \star}$ & $-0.15^{\star \star}$ & $-4.44^{\star \star}$ & $-6.59^{\star \star}$ & $-5.52^{\star \star}$ & $1.29^{\star \star}$ & $1.69^{\star \star}$ & $1.49^{\star \star}$ \\
\hline L.S.D.05 (gi) & 0.32 & 0.33 & 0.13 & 0.5 & 0.41 & 0.18 & 0.05 & 0.04 & 0.02 & 0.67 & 0.74 & 0.28 & 0.73 & 0.74 & 0.29 \\
\hline L.S.D.01 (gi) & 0.42 & 0.43 & 0.17 & 0.66 & 0.54 & 0.24 & 0.06 & 0.06 & 0.02 & 0.89 & 0.98 & 0.37 & 0.96 & 0.98 & 0.38 \\
\hline L.S.D .05(gi-gj) & 0.48 & 0.5 & 0.24 & 0.75 & 0.62 & 0.34 & 0.07 & 0.07 & 0.03 & 1.02 & 1.11 & 0.52 & 1.1 & 1.12 & 0.54 \\
\hline L.S.D .01(gi-gj) & 0.64 & 0.66 & 0.32 & 0.99 & 0.82 & 0.45 & 0.09 & 0.09 & 0.04 & 1.35 & 1.47 & 0.7 & 1.45 & 1.48 & 0.73 \\
\hline
\end{tabular}

${ }^{*}$ and ${ }^{* *}$ significant at 0.05 and 0.01 levels of probability, respectively.

$\mathrm{N}$ : normal condition. S: drought condition. Comb: combined data.

For grain yield / plant, the parental genotypes; Giza 179, GZ 28710 and IET 1444 would be the best parents for improving grain yield under normal as well as stress conditions and normal irrigation, where they gave highly significant positive estimates of $\left(\hat{g_{\mathrm{p}}}\right)$. 
In general the genotypes Giza 179, GZ 28710 and IET 1444 were the best combiners for most of the studied characters.

\section{Specific combining ability effects $\left(\overline{\mathbf{S}}_{\mathrm{ij}}^{-}\right)$:}

Estimates of specific combining ability effects $\left(\overline{\mathrm{S}}_{\mathrm{i}}\right)$ for parental combinations at the two environments and their combined data for the studied traits are given in Tables (7 and 8).

It is obvious that, eight crosses showed negative $\left(\bar{S}_{\mathrm{ij}}\right)$ for number of days to heading such estimates were maximized in GZ 1368 $\times$ IET 1444 and Sakha105 × GZ 1368. These crosses showed highly significant and negative $\left(\bar{S}_{\mathrm{ij}}\right)$ estimates which indicate that, one of these combination could be helpful for selecting early mature.

Regarding plant height, five combinations showed negative significant estimates of $\left(\bar{S}_{i j}\right)$ at both environments and their combined data. The best crosses were Sakha $105 \times$ IET 1444, Sakha $105 \times$ Wab 56 , and GZ $8710 \times$ Wab 56. These superior crosses would be considered as the desirable combinations to improve short stature in rice breeding program.

The crosses; Giza $179 \times$ Wab 56, GZ $1368 \times$ IET 1444 and Wab $56 \times$ IET 1444, were the best combinations which gave significant and positive estimates of $\left(\overline{\mathbf{S}}_{\mathrm{ij}}\right)$ for flag leaf area, chlorophyll content and relative water content under normal and stress conditions and their combined data, indicating that these crosses could be useful in breeding program. 
Table (7): Estimates of specific combining ability effects for the $F_{1}$ crosses under normal and drought conditions and their combined analysis for vegetative traits

\begin{tabular}{|c|c|c|c|c|c|c|c|c|c|c|c|c|c|c|c|}
\hline \multirow{2}{*}{ Crosses } & \multicolumn{3}{|c|}{ Days to heading (day) } & \multicolumn{3}{|c|}{ Plant Height (cm) } & \multicolumn{3}{|c|}{ Flag Leaf Area $\left(\mathrm{cm}^{2}\right)$} & \multicolumn{3}{|c|}{ Chlorophyll content $(\mathrm{mg} / \mathrm{g})$} & \multicolumn{3}{|c|}{ Relative water content } \\
\hline & $\mathbf{N}$ & $\mathbf{s}$ & Comb & $\mathbf{N}$ & $\mathbf{s}$ & Comb & $\mathbf{N}$ & $\mathbf{s}$ & Comb & $\mathbf{N}$ & $\mathbf{s}$ & Comb & $\mathbf{N}$ & $\mathbf{s}$ & Comb \\
\hline $\begin{array}{c}\text { Sakha } 102 \times \\
\text { Giza } 179\end{array}$ & $-8.11^{\star \star}$ & $-9.68^{\star \star}$ & $-8.89^{\star \star}$ & 0.8 & $4.83^{\star \star}$ & $2.81^{\star \star}$ & $-3.37^{\star \star}$ & $-4.49^{\star \star}$ & $-3.93^{\star \star}$ & -1.16 & -1.87 & $-1.52^{\star}$ & $-1.83^{\star}$ & $-2.19^{\star \star}$ & $-2.01^{\star \star}$ \\
\hline $\begin{array}{c}\text { Sakha } 102 \times \\
\text { Sakha } 105\end{array}$ & -2.36 & -0.94 & -1.65 & $5.05^{\star \star}$ & 2.48 & $3.77^{\star \star}$ & -0.71 & $2.22^{\star \star}$ & $0.75^{\star}$ & -0.69 & 0.79 & 0.05 & $3.27^{\star \star}$ & $3.49^{\star \star}$ & $3.38^{\star \star}$ \\
\hline $\begin{array}{c}\text { Sakha } 102 \times \\
\text { Sakha } 106\end{array}$ & -2.1 & 0.74 & -0.68 & 1.19 & 0.35 & 0.77 & -0.18 & 0.48 & 0.15 & 1.76 & $4.51^{\star \star}$ & $3.13^{\star \star}$ & $2.33^{\star \star}$ & $2.61^{\star \star}$ & $2.47^{\star \star}$ \\
\hline $\begin{array}{c}\text { Sakha } 102 \times \\
\text { GZ } 8710\end{array}$ & $-5.25^{\star \star}$ & $-5.35^{\star \star}$ & $-5.3^{\star \star}$ & $3.63^{\star \star}$ & -0.23 & 1.7 & -0.21 & -0.25 & -0.23 & 1.04 & 1.55 & $1.29^{\star}$ & $-4.4^{\star \star}$ & $-2.19^{\star \star}$ & $-3.29^{\star \star}$ \\
\hline $\begin{array}{c}\text { Sakha } 102 \times \\
\text { GZ } 1368\end{array}$ & $12.03^{\star \star}$ & $11.7^{\star \star}$ & $11.86^{\star \star}$ & $-5.55^{\star \star}$ & $-3.1^{\star \star}$ & $-4.32^{\star \star}$ & 0.64 & $-1.43^{\star \star}$ & -0.4 & -0.25 & 0.57 & 0.16 & $-7.53^{\star \star}$ & $-7.69^{\star \star}$ & $-7.61^{\star \star}$ \\
\hline $\begin{array}{c}\text { Sakha } 102 \times \\
\text { Wab } 56\end{array}$ & $11.01^{\star \star}$ & $10.6^{\star \star}$ & $10.81^{\star \star}$ & 2.43 & $4.14^{\star \star}$ & $3.29^{\star \star}$ & $2.17^{\star \star}$ & $4.44^{\star \star}$ & $3.3^{\star \star}$ & $-4.65^{\star \star}$ & $-3.91^{\star \star}$ & $-4.28^{\star \star}$ & 0.43 & -0.32 & 0.06 \\
\hline $\begin{array}{l}\text { Sakha } 102 \times \\
\text { IET } 1444\end{array}$ & $14.47^{\star \star}$ & $16.59^{\star \star}$ & $15.53^{\star \star}$ & -0.52 & $3.66^{\star \star}$ & 1.57 & $1.48^{\star \star}$ & $3.22^{\star \star}$ & $2.35^{\star \star}$ & -1.62 & $-2.25^{\star}$ & $-1.93^{\star \star}$ & $-1.59^{\star}$ & 0.9 & -0.35 \\
\hline $\begin{array}{l}\text { Giza 179 x } \\
\text { Sakha 105 }\end{array}$ & $15.15^{\star \star}$ & $12.09^{\star \star}$ & $13.62^{\star \star}$ & -1.65 & 1.27 & -0.19 & $2.13^{\star \star}$ & $1.78^{\star \star}$ & $1.95^{\star \star}$ & $-2.8^{\star \star}$ & $-2.47^{\star}$ & $-2.64^{\star \star}$ & -0.25 & -0.76 & -0.5 \\
\hline $\begin{array}{l}\text { Giza } 179 x \\
\text { Sakha } 106\end{array}$ & $17^{\star \star}$ & $13.72^{\star \star}$ & $15.36^{\star \star}$ & -0.4 & 0.35 & -0.03 & -0.99 & $1.16^{\star}$ & 0.08 & -1.41 & -1.77 & $-1.59^{\star}$ & $-3.43^{\star \star}$ & -1.31 & $-2.37^{\star \star}$ \\
\hline $\begin{array}{c}\text { Giza } 179 \times \text { GZ } \\
8710\end{array}$ & $15.81^{\star \star}$ & $14.32^{\star \star}$ & $15.07^{\star \star}$ & $2.79^{\star}$ & $3.19^{\star}$ & $2.99^{\star \star}$ & $1.74^{\star \star}$ & $2.08^{\star \star}$ & $1.91^{\star \star}$ & $4.3^{\star \star}$ & $2.23^{\star}$ & $3.26^{\star \star}$ & 0.25 & $-2.12^{\star \star}$ & -0.93 \\
\hline $\begin{array}{c}\text { Giza } 179 \times \text { GZ } \\
1368\end{array}$ & $4.63^{\star \star}$ & $4.22^{\star \star}$ & $4.43^{\star \star}$ & 2.42 & $3.64^{\star \star}$ & $3.03^{\star \star}$ & $3.88^{\star \star}$ & $1.12^{\star}$ & $2.5^{\star \star}$ & $-3.36^{\star \star}$ & -1.6 & $-2.48^{\star \star}$ & 0.35 & $-1.6^{\star}$ & -0.62 \\
\hline $\begin{array}{c}\text { Giza } 179 \times \\
\text { Wab } 56\end{array}$ & 1.93 & $3.22^{\star}$ & $2.57^{\star}$ & $-3.47^{\star \star}$ & $-3.78^{\star \star}$ & $-3.62^{\star \star}$ & $3.41^{\star \star}$ & $1.41^{\star \star}$ & $2.41^{\star \star}$ & $3.02^{\star \star}$ & $2.07^{\star}$ & $2.55^{\star \star}$ & $7.83^{\star \star}$ & $2.43^{\star \star}$ & $5.13^{\star \star}$ \\
\hline $\begin{array}{l}\text { Giza } 179 \times \\
\text { IET } 1444\end{array}$ & $-5.45^{\star \star}$ & $4.16^{\star \star}$ & -0.65 & $-3.87^{\star \star}$ & 1.26 & -1.31 & -0.51 & $-1.87^{\star \star}$ & $-1.19^{\star \star}$ & 0.07 & 0.78 & 0.43 & 1.29 & 1.21 & $1.25^{\star}$ \\
\hline $\begin{array}{c}\text { Sakha } 105 \times \\
\text { Sakha } 106\end{array}$ & $-3.18^{\star}$ & -0.91 & $-2.04^{\star}$ & 2.48 & -1.54 & 0.47 & $-1.39^{\star \star}$ & -0.59 & $-0.99^{\star \star}$ & 0.21 & -0.35 & -0.07 & $1.84^{\star \star}$ & $1.7^{\star}$ & $1.77^{\star \star}$ \\
\hline $\begin{array}{c}\text { Sakha } 105 \times \\
\text { GZ } 8710\end{array}$ & $-3.55^{\star}$ & -0.7 & $-2.13^{\star}$ & $-3.54^{\star}$ & -1.85 & $-2.7^{\star \star}$ & $-1.18^{\star}$ & $-1.94^{\star \star}$ & $-1.56^{\star \star}$ & $-2.62^{\star \star}$ & $-2.2^{\star}$ & $-2.41^{\star \star}$ & $3.69^{\star \star}$ & $2.6^{\star \star}$ & $3.14^{\star \star}$ \\
\hline $\begin{array}{c}\text { Sakha } 105 \times \\
\text { GZ } 1368\end{array}$ & $\overline{-}-\overline{11.27^{\star \star}}$ & $-5.85^{\star \star}$ & $-8.56^{\star \star}$ & -1.29 & -0.5 & -0.89 & 0.25 & 0.77 & 0.51 & 1.08 & -0.69 & 0.19 & $-4.11^{\star \star}$ & $-5.43^{\star \star}$ & $-4.77^{\star \star}$ \\
\hline $\begin{array}{c}\text { Sakha } 105 \times \\
\text { Wab } 56 \\
\end{array}$ & $-5.43^{\star \star}$ & $-8.07^{\star \star}$ & $-6.75^{\star \star}$ & $-8.05^{\star \star}$ & $-2.9^{\star}$ & $-5.48^{\star \star}$ & $-2.11^{\star \star}$ & $-1.33^{\star}$ & $-1.72^{\star \star}$ & 0.82 & 1.52 & 1.17 & $-5.62^{\star \star}$ & $-7.67^{\star \star}$ & $-6.64^{\star \star}$ \\
\hline $\begin{array}{l}\text { Sakha } 105 \times \\
\text { IET } 1444\end{array}$ & $12.47^{\star \star}$ & $12.76^{\star \star}$ & $12.62^{\star \star}$ & $-7.88^{\star \star}$ & $-4.57^{\star \star}$ & $-6.22^{\star \star}$ & 0.03 & $-1.74^{\star \star}$ & $-0.85^{\star}$ & 0.26 & -0.84 & -0.29 & $-3.93^{\star \star}$ & 0.32 & $-1.81^{\star \star}$ \\
\hline $\begin{array}{c}\text { Sakha } 106 \times \\
\text { GZ } 8710\end{array}$ & -1.46 & -0.78 & -1.12 & -0.63 & 1.93 & 0.65 & $1.96^{\star \star}$ & $3.74^{\star \star}$ & $2.85^{\star \star}$ & 0.39 & $2.08^{\star}$ & 1.23 & $-4.89^{\star \star}$ & $-2.73^{\star \star}$ & $-3.81^{\star \star}$ \\
\hline $\begin{array}{c}\text { Sakha } 106 \times \\
\text { GZ } 1368\end{array}$ & -0.62 & -0.53 & -0.58 & -0.59 & $3.84^{\star \star}$ & 1.63 & 0.29 & $-1.88^{\star \star}$ & $-0.8^{\star}$ & 0.9 & $-4.39^{\star \star}$ & $-1.75^{\star \star}$ & $-1.48^{\star}$ & -1.32 & $-1.4^{\star \star}$ \\
\hline $\begin{array}{c}\text { Sakha } 106 \times \\
\text { Wab } 56\end{array}$ & $3.48^{\star}$ & $5.27^{\star \star}$ & $4.38^{\star \star}$ & $-3.18^{\star}$ & 1.26 & -0.96 & $-1.65^{\star \star}$ & $-2.23^{\star \star}$ & $-1.94^{\star \star}$ & $-4.19^{\star \star}$ & $-2.47^{\star}$ & $-3.33^{\star \star}$ & $3.98^{\star \star}$ & $3.84^{\star \star}$ & $3.91^{\star \star}$ \\
\hline $\begin{array}{c}\text { Sakha } 106 \times x \\
\text { IET } 1444\end{array}$ & $-6.89^{\star \star}$ & $-6.86^{\star \star}$ & $-6.88^{\star \star}$ & 0.64 & -1.58 & -0.47 & $1.1^{\star}$ & $2.59^{\star \star}$ & $1.85^{\star \star}$ & $-2.4^{\star \star}$ & -0.94 & $-1.67^{\star}$ & $-2.17^{\star \star}$ & $-4.06^{\star \star}$ & $-3.12^{\star \star}$ \\
\hline $\begin{array}{c}\text { GZ } 8710 \times \text { GZ } \\
1368\end{array}$ & $7.1^{\star \star}$ & $7.25^{\star \star}$ & $7.17^{\star \star}$ & $-9.17^{\star \star}$ & $2.79^{\star}$ & $-3.19^{\star \star}$ & 0.47 & $1.55^{\star \star}$ & $1.01^{\star \star}$ & $-2.93^{\star \star}$ & -1.33 & $-2.13^{\star \star}$ & 0.89 & $-3.41^{\star \star}$ & $-1.26^{\star}$ \\
\hline $\begin{array}{c}\text { GZ } 8710 \times \\
\text { Wab 56 }\end{array}$ & -0.96 & -0.87 & -0.91 & $-2.89^{\star}$ & $-5.89^{\star \star}$ & $-4.39^{\star \star}$ & 0.86 & 0.05 & 0.46 & $-3.92^{\star \star}$ & $-2.17^{\star}$ & $-3.05^{\star \star}$ & $-1.7^{\star}$ & 0.26 & -0.72 \\
\hline $\begin{array}{c}\text { GZ } 8710 \times \text { IET } \\
1444\end{array}$ & $-3.05^{\star}$ & $7.84^{\star \star}$ & $2.39^{\star}$ & $-3.1^{*}$ & 1.98 & -0.56 & -0.16 & $-1.35^{\star}$ & $-0.76^{\star}$ & $-4.28^{\star \star}$ & -1.28 & $-2.78^{\star \star}$ & $-1.8^{\star}$ & $-2.74^{\star \star}$ & $-2.27^{\star \star}$ \\
\hline $\begin{array}{c}\text { GZ } 1368 \times \\
\text { Wab 56 }\end{array}$ & $-6.53^{\star \star}$ & $-5.89^{\star \star}$ & $-6.21^{\star \star}$ & $4.61^{\star \star}$ & 1.16 & $2.88^{\star \star}$ & 0.41 & $1.32^{\star}$ & $0.86^{\star}$ & -0.12 & -1.88 & -1 & $3.12^{\star \star}$ & $6.1^{\star \star}$ & $4.61^{\star \star}$ \\
\hline $\begin{array}{c}\text { GZ } 1368 \times \text { IET } \\
1444 \\
\end{array}$ & $14 . \overline{7} 1^{\star \star}$ & $12 . \overline{2} 8^{\star \star}$ & $-13.5^{\star \star}$ & $12.17^{\star \star}$ & $6.47^{\star \star}$ & $9.32^{\star \star}$ & $3.74^{\star \star}$ & $2.31^{\star \star}$ & $3.03^{\star \star}$ & $4.5^{\star \star}$ & $4.6^{\star \star}$ & $4.55^{\star \star}$ & $4.84^{\star \star}$ & $3.58^{\star \star}$ & $4.21^{\star \star}$ \\
\hline $\begin{array}{c}\text { Wab } 56 \times \text { IET } \\
1444\end{array}$ & $-4.59^{\star \star}$ & $-3.87^{\star \star}$ & $-4.23^{\star \star}$ & $5.56^{\star \star}$ & $4.25^{\star \star}$ & $4.91^{\star \star}$ & $2.01^{\star \star}$ & $2.04^{\star \star}$ & $2.02^{\star \star}$ & $4.31^{\star \star}$ & $4.7^{\star \star}$ & $4.5^{\star \star}$ & $5.62^{\star \star}$ & $6.13^{\star \star}$ & $5.87^{\star \star}$ \\
\hline L.S.D.05(sij) & 2.80 & 2.77 & 1.94 & 2.71 & 2.66 & 1.86 & 1.01 & 1.06 & 0.72 & 1.78 & 1.93 & 1.29 & 1.39 & 1.49 & 1.00 \\
\hline L.S.D.01(sij) & 3.71 & 3.67 & 2.58 & 3.58 & 3.52 & 2.48 & 1.34 & 1.40 & 0.96 & 2.35 & 2.55 & 1.72 & 1.84 & 1.98 & 1.34 \\
\hline $\begin{array}{l}\text { L.S.D.05(sij- } \\
\text { sik) }\end{array}$ & 4.15 & 4.11 & 2.87 & 4.00 & 3.93 & 2.76 & 1.50 & 1.56 & 1.06 & 2.63 & 2.85 & 1.91 & 2.06 & 2.21 & 1.48 \\
\hline $\begin{array}{c}\text { L.S.D.01(sij- } \\
\text { sik) }\end{array}$ & 5.49 & 5.43 & 3.82 & 5.30 & 5.20 & 3.67 & 1.98 & 2.07 & 1.42 & 3.48 & 3.78 & 2.54 & 2.72 & 2.92 & 1.98 \\
\hline $\begin{array}{l}\text { L.S.D.05(sij- } \\
\text { skl) }\end{array}$ & 3.91 & 3.87 & 0.96 & 3.78 & 3.71 & 0.92 & 1.41 & 1.47 & 0.35 & 2.48 & 2.69 & 0.64 & 1.94 & 2.08 & 0.49 \\
\hline $\begin{array}{c}\text { L.S.D.01(sij- } \\
\text { skl) }\end{array}$ & 5.17 & 5.12 & 1.27 & 5.00 & 4.90 & 1.22 & 1.87 & 1.95 & 0.47 & 3.28 & 3.56 & 0.85 & 2.57 & 2.76 & 0.66 \\
\hline
\end{tabular}

For panicle length, eight out of twenty eight hybrid combinations showed highly significant and positive $\left(\bar{S}_{\mathrm{i}}\right)$ under both environments and their combined data. The best crosses which showed desirable values under drought stress were; Sakha $102 \times$ Sakha 106, Sakha 102 
$\times$ GZ 8710, Giza $179 \times$ GZ 8710, GZ $1368 \times$ IET1444 and Wab $56 \times$ IET 1444.

For number of panicles/plant, eleven out of the twenty eight crosses showed highly significant positive $\left(\bar{S}_{i j}\right)$ at both environments and their combined data. The crosses which had the highest values were Giza 179 × Sakha 105, GZ $1368 \times$ Wab56, and Sakha $106 \times$ GZ 1368 where they recorded the maximum $\left(\overline{\mathbf{S}}_{i j}\right)$, indicating that these crosses would be used in breeding program to improve this trait.

Concerning 100-kernel weight, seven hybrid combinations showed highly significant positive $\left(\overline{\mathrm{S}}_{\mathrm{i}}\right)$. The crosses; $\mathrm{GZ} 8710 \times \mathrm{GZ}$ 1368, GZ $8710 \times$ Wab 56 and Sakha $106 \times$ GZ 8710. The crosses which gave the highest values and significant of 100 -kernel weight could be used in breeding program for enhancing this trait.

Regarding sterility percentage, seven out of the twenty eight crosses expressed highly significant and negative $\left(\overline{\mathrm{S}}_{\mathrm{ij}}\right)$ at both condition and their combined analysis. The crosses; Sakha $102 \times$ IET 1444, Giza $179 \times$ GZ 8710 and Giza $179 \times$ Sakha 106 were the most desirable crosses for sterility percentage.

Regarding grain yield/plant, eight hybrid combinations showed highly significant and positive $\left(\overline{\mathbf{S}}_{i j}\right)$ under normal and drought conditions and their combined data. The most desirable $\left(\bar{S}_{\mathrm{ij}}\right)$ were detected in the Giza $179 \times$ Sakha 106, Giza $179 \times$ Sakha 105, Giza $179 \times$ GZ 8710 and Sakha $102 \times$ IET 1444. These hybrid combinations showed desirable $\left(\overline{\mathrm{S}}_{\mathrm{i}}\right)$ for most yield components and high 
Table (8): Estimates of specific combining ability effects for the $F_{1}$ crosses under normal and drought conditions and their combined analysis for grain yield and its components traits

\begin{tabular}{|c|c|c|c|c|c|c|c|c|c|c|c|c|c|c|c|}
\hline \multirow{2}{*}{ Crosses } & \multicolumn{3}{|c|}{ Panicle length $(\mathrm{cm})$} & \multicolumn{3}{|c|}{ No of panicles / plant } & \multicolumn{3}{|c|}{ 100-kernel weight $(\mathrm{g})$} & \multicolumn{3}{|c|}{ Sterility \% } & \multicolumn{3}{|c|}{ Grain yield/plant $(\mathrm{g})$} \\
\hline & $\mathbf{N}$ & s & Comb & $\mathbf{N}$ & s & Comb & $\mathbf{N}$ & $\mathbf{s}$ & Comb & $\mathbf{N}$ & s & Comb & N & s & Comb \\
\hline $\begin{array}{c}\text { Sakha } 102 \times \\
\text { Giza } 179 \\
\end{array}$ & $2.97^{\star \star}$ & $-1.5^{\star \star}$ & $0.74^{\star}$ & $-6.68^{\star \star}$ & $-1.95^{\star \star}$ & $-4.31^{\star \star}$ & $0.26^{\star \star}$ & $0.24^{\star \star}$ & $0.25^{\star \star}$ & $15.74^{\star \star}$ & $18.95^{\star \star}$ & $17.34^{\star \star}$ & $-7.28^{\star \star}$ & $-5.8^{\star \star}$ & $-6.54^{\star \star}$ \\
\hline $\begin{array}{l}\text { Sakha } 102 \times \\
\text { Sakha } 105\end{array}$ & -0.88 & -0.07 & -0.48 & $-2.68^{\star \star}$ & -0.72 & $-1.7^{\star \star}$ & $-0.18^{\star}$ & 0.01 & -0.08 & $13.45^{\star \star}$ & $10.51^{\star \star}$ & $11.98^{\star \star}$ & $-2.9^{\star}$ & -1.64 & $-2.27^{\star \star}$ \\
\hline $\begin{array}{l}\text { Sakha } 102 \times \\
\text { Sakha } 106\end{array}$ & $1.02^{\star}$ & $2.39^{\star \star}$ & $1.7^{\star \star}$ & $-3.54^{\star \star}$ & 0.19 & $-1.68^{\star \star}$ & 0.08 & $0.18^{\star \star}$ & $0.13^{\star \star}$ & $16.83^{\star \star}$ & $17.94^{\star \star}$ & $17.38^{\star \star}$ & 1.79 & 1.04 & 1.42 \\
\hline $\begin{array}{c}\text { Sakha } 102 \times \text { GZ } \\
8710\end{array}$ & $1.71^{\star \star}$ & $1.37^{\star \star}$ & $1.54^{\star \star}$ & $-2.78^{\star \star}$ & 0.05 & $-1.37^{\star \star}$ & 0.1 & $-0.38^{\star \star}$ & $-0.14^{\star \star}$ & $13.31^{\star \star}$ & $17.52^{\star \star}$ & $15.41^{\star \star}$ & $-2.9^{\star}$ & 2.05 & -0.43 \\
\hline $\begin{array}{c}\text { Sakha } 102 \times \text { GZ } \\
1368\end{array}$ & $1^{*}$ & $2.09^{\star \star}$ & $1.55^{\star \star}$ & $3.78^{\star \star}$ & $2.36^{\star \star}$ & $3.07^{\star \star}$ & 0.03 & -0.01 & 0.01 & $7.95^{\star \star}$ & $10.65^{\star \star}$ & $9.3^{* *}$ & $2.72^{\star}$ & 2.02 & $2.37^{\star \star}$ \\
\hline $\begin{array}{c}\text { Sakha } 102 \times \\
\text { Wab } 56\end{array}$ & 0.8 & $1.59^{\star \star}$ & $1.19^{\star \star}$ & $4.73^{\star \star}$ & $4.47^{\star \star}$ & $4.6^{\star \star}$ & $0.17^{\star}$ & $0.18^{\star \star}$ & $0.18^{\star \star}$ & $-5.24^{\star \star}$ & $-12.21^{\star \star}$ & $-8.72^{\star \star}$ & $3.66^{\star \star}$ & $2.85^{\star}$ & $3.26^{\star \star}$ \\
\hline $\begin{array}{l}\text { Sakha } 102 \times \\
\text { IET } 1444 \\
\end{array}$ & -0.46 & 0.72 & 0.13 & $1.65^{\star}$ & $3.75^{\star \star}$ & $2.7^{\star \star}$ & -0.06 & 0.11 & 0.02 & $-14.14^{\star \star}$ & $-17.86^{\star \star}$ & $-16^{\star \star}$ & $5.75^{\star \star}$ & $4.18^{\star \star}$ & $4.96^{\star \star}$ \\
\hline $\begin{array}{l}\text { Giza } 179 \times \\
\text { Sakha } 105 \\
\end{array}$ & -0.49 & $1.53^{\star \star}$ & 0.52 & $5.44^{\star \star}$ & $5.06^{\star \star}$ & $5.25^{\star \star}$ & -0.02 & 0.1 & 0.04 & 1.94 & $9.89^{* *}$ & $5.91^{\star \star}$ & $6.14^{\star \star}$ & $5.45^{\star \star}$ & $5.79^{\star \star}$ \\
\hline $\begin{array}{l}\text { Giza } 179 \times \\
\text { Sakha } 106\end{array}$ & 0.55 & $1.57^{\star \star}$ & $1.06^{\star \star}$ & $3.02^{\star \star}$ & $1.86^{\star \star}$ & $2.44^{\star \star}$ & -0.12 & $-0.15^{\star}$ & $-0.13^{\star \star}$ & $-10.49^{\star \star}$ & $-14.6^{\star \star}$ & $-12.55^{\star \star}$ & $7.01^{\star \star}$ & $6.06^{\star \star}$ & $6.53^{\star \star}$ \\
\hline $\begin{array}{c}\text { Giza } 179 \times \text { GZ } \\
8710 \\
\end{array}$ & $1.98^{\star \star}$ & $3.61^{\star \star}$ & $2.8^{\star \star}$ & $3.03^{\star \star}$ & $1.55^{\star}$ & $2.29^{\star \star}$ & $0.16^{\star}$ & $0.25^{\star \star}$ & $0.2^{\star \star}$ & $-10.91^{\star \star}$ & $-14.22^{\star \star}$ & $-12.57^{\star \star}$ & $4.95^{\star \star}$ & $4.47^{\star \star}$ & $4.71^{\star \star}$ \\
\hline $\begin{array}{c}\text { Giza } 179 \times \text { × GZ } \\
1368\end{array}$ & $1.42^{\star \star}$ & $1.68^{\star \star}$ & $1.55^{\star \star}$ & $-4.01^{\star \star}$ & -0.15 & $-2.08^{\star \star}$ & 0.13 & 0.11 & $0.12^{\star}$ & $9.23^{\star \star}$ & $14.84^{\star \star}$ & $12.04^{\star \star}$ & $3.6^{\star \star}$ & 0.84 & $2.22^{\star \star}$ \\
\hline $\begin{array}{c}\text { Giza } 179 \times \text { Wab } \\
56 \\
\end{array}$ & 0.95 & $2.03^{\star \star}$ & $1.49^{\star \star}$ & $-2.34^{\star \star}$ & 0.57 & -0.89 & 0.01 & -0.13 & -0.06 & $5.57^{\star \star}$ & $14.04^{\star *}$ & $9.81^{\star \star}$ & -1.09 & -0.93 & -1.01 \\
\hline $\begin{array}{c}\text { Giza } 179 \times \text { IET } \\
1444\end{array}$ & -0.22 & 0.55 & 0.16 & 1.32 & $1.77^{\star \star}$ & $1.54^{\star \star}$ & $0.18^{\star}$ & -0.05 & 0.06 & $-4.05^{\star \star}$ & $-8.77^{\star \star}$ & $-6.41^{\star \star}$ & $3.07^{\star \star}$ & $3.73^{\star \star}$ & $3.4^{\star \star}$ \\
\hline $\begin{array}{c}\text { Sakha } 105 \times \\
\text { Sakha } 106\end{array}$ & 0.01 & -0.02 & -0.01 & $-3.49^{\star \star}$ & -0.54 & $-2.01^{\star \star}$ & $0.18^{\star}$ & $-0.42^{\star \star}$ & $-0.12^{\star}$ & $-3.23^{\star \star}$ & -2.09 & $-2.66^{\star \star}$ & $-3.78^{\star \star}$ & $-2.95^{*}$ & $-3.37^{\star \star}$ \\
\hline $\begin{array}{c}\text { Sakha } 105 \times \text { GZ } \\
8710\end{array}$ & $1.58^{\star \star}$ & $2.1^{\star \star}$ & $1.84^{\star \star}$ & $-2.19^{\star \star}$ & -1.02 & $-1.6^{\star \star}$ & $-0.25^{\star \star}$ & -0.14 & $-0.19^{\star \star}$ & $-5.84^{\star \star}$ & 0.77 & $-2.54^{\star \star}$ & $-3.24^{\star \star}$ & $-2.78^{\star}$ & $-3.01^{\star \star}$ \\
\hline $\begin{array}{c}\text { Sakha } 105 \times \text { GZ } \\
1368\end{array}$ & $-1.14^{\star}$ & -0.79 & $-0.97^{\star \star}$ & -0.17 & $-1.34^{\star}$ & -0.76 & -0.12 & -0.02 & -0.07 & 0.31 & $-4.93^{\star \star}$ & $-2.31^{\star \star}$ & -2.12 & -0.93 & -1.53 \\
\hline $\begin{array}{l}\text { Sakha } 105 \times \\
\text { Wab } 56\end{array}$ & $1.43^{\star \star}$ & -0.2 & 0.62 & $2.29^{\star \star}$ & $4.02^{\star \star}$ & $3.15^{\star \star}$ & -0.07 & 0.09 & 0.01 & $4.51^{\star \star}$ & 1.27 & $2.89^{\star \star}$ & $3.35^{\star \star}$ & $4.06^{\star \star}$ & $3.71^{\star \star}$ \\
\hline $\begin{array}{l}\text { Sakha } 105 \times \\
\text { IET } 1444\end{array}$ & 0.22 & $-1.07^{\star}$ & -0.43 & $3.08^{\star \star}$ & $3.36^{\star \star}$ & $3.22^{\star \star}$ & $0.18^{\star}$ & $0.22^{\star \star}$ & $0.2^{\star \star}$ & $8.8^{\star \star *}$ & $8.28^{* \star}$ & $8.54^{\star \star}$ & 0.31 & 1.39 & 0.85 \\
\hline $\begin{array}{c}\text { Sakha } 106 \times \text { GZ } \\
8710\end{array}$ & $-1.02^{\star}$ & -0.58 & $-0.8^{\star}$ & $2.58^{\star \star}$ & $4.1^{\star \star}$ & $3.34^{\star \star}$ & $0.25^{\star \star}$ & $0.33^{\star \star}$ & $0.29^{\star \star}$ & $15.07^{\star \star}$ & $2.61^{*}$ & $8.84^{\star \star}$ & 0.69 & $-2.46^{*}$ & -0.89 \\
\hline $\begin{array}{c}\text { Sakha } 106 \times \text { GZ } \\
1368\end{array}$ & $-1.09^{\star}$ & $-1.75^{\star \star}$ & $-1.42^{\star \star}$ & $-4.46^{\star \star}$ & $-3.53^{\star \star}$ & $-4^{\star \star}$ & 0.09 & 0.03 & 0.06 & 1.7 & $5.59^{\star \star}$ & $3.64^{\star \star}$ & $-3.06^{\star \star}$ & $-2.32^{\star}$ & $-2.69^{\star \star}$ \\
\hline $\begin{array}{c}\begin{array}{l}\text { Sakha } 106 \times \\
\text { Wab } 56\end{array} \\
\end{array}$ & -0.61 & $-1.14^{\star}$ & $-0.87^{\star}$ & $-3.57^{\star \star}$ & $-1.88^{\star \star}$ & $-2.72^{\star \star}$ & 0.09 & -0.09 & 0.002 & $9.47^{\star \star}$ & $13.62^{\star \star}$ & $11.54^{\star \star}$ & 1.68 & -0.99 & 0.35 \\
\hline $\begin{array}{l}\text { Sakha } 106 \times x \\
\text { IET } 1444\end{array}$ & $1.81^{\star \star}$ & -0.01 & $0.9^{*}$ & $4.23^{\star \star}$ & $1.79^{\star \star}$ & $3.01^{\star \star}$ & $-0.64^{\star \star}$ & 0.08 & $-0.28^{\star \star}$ & $-7.87^{\star \star}$ & $-7.94^{\star \star}$ & $-7.91^{\star \star}$ & 1.91 & $5.34^{\star \star}$ & $3.62^{\star \star}$ \\
\hline $\begin{array}{c}\text { GZ } 8710 \times \text { GZ } \\
1368 \\
\end{array}$ & $-2.87^{\star \star}$ & $-2.1^{\star \star}$ & $-2.49^{\star *}$ & $2.25^{\star \star}$ & $1.43^{\star}$ & $1.84^{\star \star}$ & $0.19^{\star \star}$ & $0.46^{\star \star}$ & $0.32^{\star \star}$ & 0.33 & $-2.87^{\star}$ & -1.27 & 2.18 & 0.19 & 1.18 \\
\hline $\begin{array}{c}\text { GZ } 8710 \times \text { Wab } \\
56\end{array}$ & $-1.74^{\star \star}$ & -0.62 & $-1.18^{\star \star}$ & 0.41 & -0.42 & -0.01 & $0.22^{\star \star}$ & $0.37^{\star \star}$ & $0.3^{\star \star}$ & $3.77^{\star \star}$ & $5.52^{* *}$ & $4.65^{\star \star}$ & $2.55^{\star}$ & $-2.68^{\star}$ & -0.06 \\
\hline $\begin{array}{c}\text { GZ } 8710 \times \text { IET } \\
1444\end{array}$ & $-2.02^{\star \star}$ & $-1.64^{\star \star}$ & $-1.83^{\star \star}$ & $-2.55^{\star \star}$ & $-1.52^{\star}$ & $-2.03^{\star \star}$ & $0.2^{\star \star}$ & -0.08 & 0.06 & $5.86^{\star \star}$ & $7.95^{\star *}$ & $6.91^{\star \star}$ & $4.38^{\star \star}$ & -0.06 & $2.16^{\star \star}$ \\
\hline $\begin{array}{c}\text { GZ } 1368 \times \text { Wab } \\
56\end{array}$ & $1.19^{\star}$ & $3.25^{\star \star}$ & $2.22^{\star \star}$ & $5.09^{\star \star}$ & $4.06^{\star \star}$ & $4.57^{\star \star}$ & 0.01 & 0.02 & 0.01 & $-9.66^{\star \star}$ & $-13.55^{\star \star}$ & $-11.6^{\star \star}$ & $8.47^{\star \star}$ & $3.63^{\star \star}$ & $6.05^{\star \star}$ \\
\hline $\begin{array}{c}\text { GZ } 1368 \times \text { IET } \\
1444\end{array}$ & $2.16^{\star \star}$ & $3.07^{\star \star}$ & $2.61^{\star \star}$ & $-5.28^{\star \star}$ & $-4.16^{\star \star}$ & $-4.72^{\star \star}$ & 0.07 & $-0.4^{\star \star}$ & $-0.16^{\star \star}$ & 1.38 & $3.52^{\star \star *}$ & $2.45^{\star \star}$ & -0.27 & 0.52 & 0.12 \\
\hline $\begin{array}{c}\text { Wab } 56 \times \text { IET } \\
1444\end{array}$ & $2.04^{\star \star}$ & $2.79^{\star \star}$ & $2.42^{\star \star}$ & $-5.21^{\star \star}$ & $-4.38^{\star \star}$ & $-4.8^{\star \star}$ & -0.01 & $-0.31^{\star \star}$ & $-0.16^{\star \star}$ & $5.85^{\star \star}$ & $11.4^{\star \star}$ & $8.63^{\star \star}$ & -0.59 & -1.29 & -0.94 \\
\hline L.S.D.05(sij) & 0.97 & 1.01 & 0.69 & 1.52 & 1.26 & 0.97 & 0.14 & 0.14 & 0.1 & 2.07 & 2.26 & 1.5 & 2.23 & 2.26 & 1.56 \\
\hline L.S.D.01(sij) & 1.29 & 1.33 & 0.92 & 2.01 & 1.66 & 1.29 & 0.19 & 0.18 & 0.13 & 2.73 & 2.99 & 2 & 2.95 & 3 & 2.08 \\
\hline $\begin{array}{l}\text { L.S.D.05(sij- } \\
\text { sik) }\end{array}$ & 1.44 & 1.49 & 1.02 & 2.25 & 1.86 & 1.43 & 0.21 & 0.2 & 0.14 & 3.06 & 3.34 & 2.23 & 3.3 & 3.35 & 2.31 \\
\hline $\begin{array}{l}\text { L.S.D.01(sij- } \\
\text { sik) }\end{array}$ & 1.91 & 1.97 & 1.36 & 2.98 & 2.46 & 1.91 & 0.28 & 0.27 & 0.19 & 4.05 & 4.42 & 2.96 & 4.36 & 4.43 & 3.08 \\
\hline $\begin{array}{l}\text { L.S.D.05(sij- } \\
\text { skl) }\end{array}$ & 1.36 & 1.4 & 0.34 & 2.12 & 1.75 & 0.48 & 0.2 & 0.19 & 0.05 & 2.88 & 3.15 & 0.74 & 3.11 & 3.16 & 0.77 \\
\hline $\begin{array}{l}\text { L.S.D.01(sij- } \\
\text { skl) }\end{array}$ & 1.8 & 1.86 & 0.45 & 2.81 & 2.32 & 0.64 & 0.26 & 0.25 & 0.06 & 3.81 & 4.17 & 0.99 & 4.11 & 4.18 & 1.03 \\
\hline
\end{tabular}

${ }^{*}$ and ${ }^{* *}$ significant at 0.05 and 0.01 levels of probability, respectively.

$\mathrm{N}$ : normal condition. S: drought condition. Comb: combined data. 
mean performance. However, the cross combinations; Giza $179 \times \mathrm{GZ}$ 8710 and Sakha $102 \times$ Wab 56 had significant $\left(\bar{S}_{i j}\right)$ in desirable direction for grain yield/plant and their components i.e., panicle length, no. of panicles/plant, 100-kernel weight and sterility percentage. This might indicate that, these hybrid combination could be used in improving grain yield at normal as well as stress irrigation regimes under the situation of the present investigation. Therefore, these superior crosses would be of practical interest in breeding program for developing rice varieties under drought conditions.

\section{REFERENCES}

Abd Allah, A.A. (2004).A breeding study on drought tolerance in rice (Orayza sativa L.). Egyptian J. of Agric., Res., 82(1):149-165.

Abd El-Hadi, A.H.; H.F. El-Mowafi and E.A. Ramadan (2014). Genetic analysis for yield and its components traits under water stress condition in rice (Oryza Sativa L.). J. Agric., Chem., and Biotechn., Mansoura Univ., 5(1): 1-12.

Abd El-Lateef A.S.; A.B. El-Abd and A.A. Abdallah (2006). Genetic studies of rice root characters related to drought tolerance. The first field crops conference program and abstracts 22-24 August, Giza, Egypt, (abstract).

Araus, J.L.; G.A. Slafer; M.P. Reynolds and C. Royo (2002). Plant breeding and relations ing3 Cereals: what to breed for? Annals of Botany., 89:925-940.

Bartlett, M.M. (1937).Properties of sufficiency and statistical tests. Proc., Roy., Soc., London, series A, 160:268-282.

Bastawisi A.O.; H.F. El-Mowafi; M.A. Maximos and M.F. Sobaa (2005).Hybrid rice production technology in Egypt. In: Proceeding: workshop on Rice Integrated Crop Management Systems for Food Security in the Near East Countries, Alexandria, Egypt, 27-29 July 2003. Food and Agriculture Organization of the United Nations Regional Office for the Near East, Cairo.

Bouman, B.A.M.; S. Peng; A.R. Castaoeda and R.M. Visperas (2005). Yield and water use irrigated tropical aerobic rice systems. Agriculture water management, 74(2):87-105.

El-Diasty, Z.M.; H.F. El-Mowafi; M.S. Hamada and R.M. Abdallah (2008). Genetic studies on Photo-thermo-sensitive genic male sterility (P/TGMS) and its utilization in rice breeding. J. Agric., Sci., Mansoura Univ., 33(5): 3391-3404.

El- Hity, M.A.; M.S. Abd El-Aty; A.A. Hadifa and M. Abo.Omar (2015).Combining Ability for Yield And Some Agronomic Characters as Indices of Drought Tolerance in Rice (Oryza Sativa L.).J. Agric. Sci., kafrelsheikh Univ., 32(3): 773-795. 
El-Mowafi, H.F. and A.A. Abou-Shousha (2003).Combining ability and heterosis analysis of diverse CMS lines in hybrid rice. J. Agric., Res., Tanta Univ., 29(1): 106-127.

Gaballa, M.M. (2009). Studies on physiological and morphological traits associated with drought resistance in rice (Oryza sativa L.). phD. Thesis, Fac., Agric., Kafr El-sheikh Univ., Egypt.

Griffing, B. (1956).Concept of general and specific combining ability in relation to diallel crossing system.Austr., J. Bio., Sci., 9:463498.

Hadifa, A. AM. (2012).The role of root and shoot characteristics in drought tolerance. PhD. Thesis.Fac., of agric., Kafr El-Sheikh Univ., Kafr El-Sheikh, Egypt.

Khush, G.S. (2005). What it will take to feed? 5 billion rice consumers by 2030. Plant Mol., Biol., 59:1-6.

Khush, G.S. and D.S. Brar (2002). Biotechnology for rice breeding: progress and potential impact. Sustainable rice production for food security.Proceed 20th Session Int. Rice Comm. pp. 23-26 July. Bangkok, Thailand.

Mirarab, M.; A. Ahmadikhah and M.H. Pahlavani (2011).Study on combining ability, heterosis and genetic parameters of yield traits in rice. African Journal of Biotechnology. 10(59):1251212519.

Mostajeran, A. and V. Rahimi-Eichi (2009).Effect of drought stress on growth and yield of rice (Oryza sativa L.). Cultivars and accumulation of proline and soluble sugars in sheath and blades of their different ages leaves. 5(2): 264-272.

Steel, R.G. and J.H. Torrie (1980). Principles and procedures of statistics. $2^{\text {th }}$ ed. McGraw-Hill Book Company, New york, USA.

Venuprasad, R.; H.R. Lafitte and G.N. Atlin (2007).Response to direct selection for grain yield under drought stress in Rice.Crop. Sci., 47:285-293.

Yan, S.; S. Wen; Z. Li; J. Wan; Y. Tian and C. Liu (2000).Study on the combining ability of indica two-line hybrid rice. Journal of Huazhang Agriculture University 19:204-208.

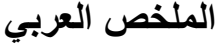

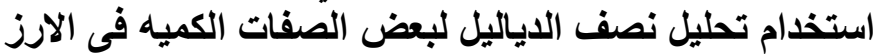

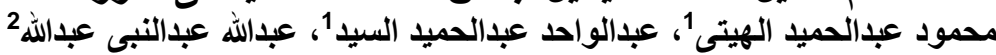

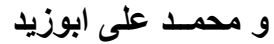

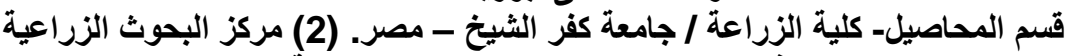

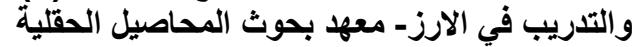

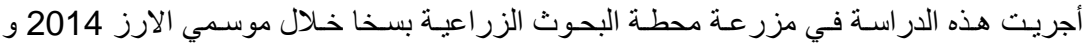

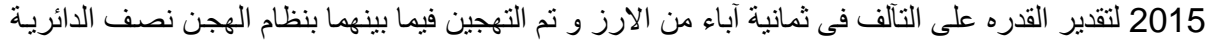

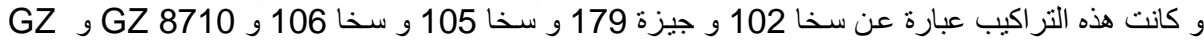

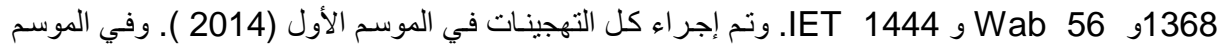

$$
\begin{aligned}
& \text { الثاني } 2015 \text { تمت زر اعة الآباء الثمانية و هجنها الثمانية و العشرين تحت معاملتي ري في تجربتين منفصلتين، التين }
\end{aligned}
$$




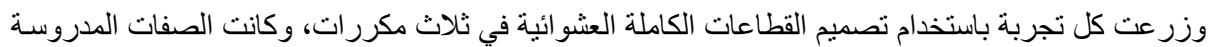

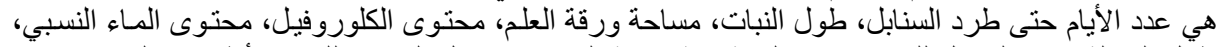

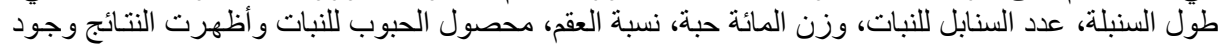

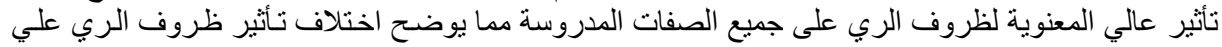

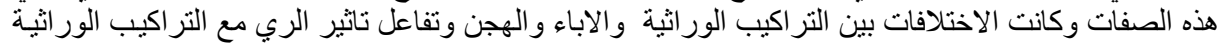

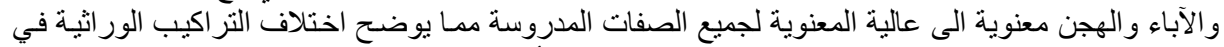

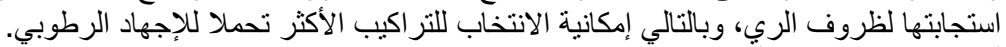

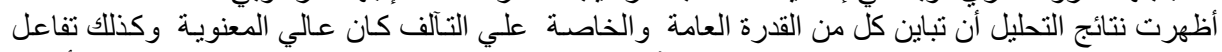

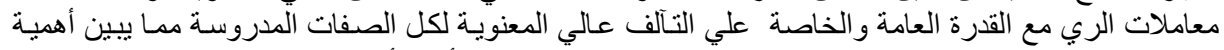

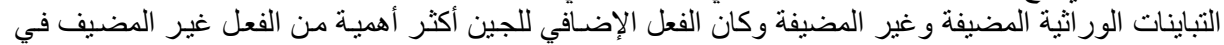
وراثة هذه الصفات.

كانت التر اكيب الور اثية الأبوية جيزة 179، GZ8710 ذات قدره ائتلافيه عالية لصفات الصات عدد السنابل بالنبات،

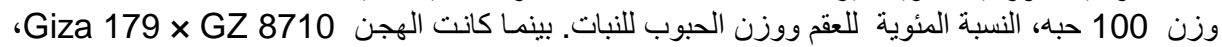
Sakha 102 × IET 1444 Giza 179 × Sakha 106 ، GZ 1368 × IET 1444 ايتتلافيه خاصه عالية لصفات المحصول ومعظم مكوناته تحت كلا من ظروف الري العادية وظروف الاجهاد 